\title{
COVID-19 Severity Prediction Using Enhanced Whale with Salp Swarm Feature Classification
}

\author{
Nebojsa Budimirovic ${ }^{1}$, E. Prabhu ${ }^{2}$, Milos Antonijevic ${ }^{1}$, Miodrag Zivkovic ${ }^{1}$, Nebojsa Bacanin ${ }^{1, *}$, \\ Ivana Strumberger ${ }^{1}$ and K. Venkatachalam ${ }^{3}$
}

\author{
${ }^{1}$ Singidunum University, Belgrade, 11000, Serbia \\ ${ }^{2}$ Department of Electronics and Communication Engineering, Amrita School of Engineering, Amrita Vishwa \\ Vidyapeetham, Coimbatore, 641112, India \\ ${ }^{3}$ Department of Applied Cybernetics, Faculty of Science, University of Hradec Králové, Hradec Králové, 50003, Czech \\ Republic \\ *Corresponding Author: Nebojsa Bacanin. Email: nbacanin@singidunum.ac.rs \\ Received: 07 September 2021; Accepted: 06 January 2022
}

\begin{abstract}
Computerized tomography (CT) scans and X-rays play an important role in the diagnosis of COVID-19 and pneumonia. On the basis of the image analysis results of chest CT and X-rays, the severity of lung infection is monitored using a tool. Many researchers have done in diagnosis of lung infection in an accurate and efficient takes lot of time and inefficient. To overcome these issues, our proposed study implements four cascaded stages. First, for pre-processing, a mean filter is used. Second, texture feature extraction uses principal component analysis (PCA). Third, a modified whale optimization algorithm is used (MWOA) for a feature selection algorithm. The severity of lung infection is detected on the basis of age group. Fourth, image classification is done by using the proposed MWOA with the salp swarm algorithm (MWOA-SSA). MWOA-SSA has an accuracy of 97\%, whereas PCA and MWOA have accuracies of $81 \%$ and $86 \%$. The sensitivity rate of the MWOA-SSA algorithm is better that of than PCA (84.4\%) and MWOA $(95.2 \%)$. MWOA-SSA outperforms other algorithms with a specificity of $97.8 \%$. This proposed method improves the effective classification of lung affected images from large datasets.
\end{abstract}

Keywords: PCA; WOA; CT-image; lung infection; COVID-19

\section{Introduction}

COVID-19 is a virus infection that has changed human life in various aspects including finance, education, health care, and supply chains. People with COVID-19 infection are facing respiratory problems and can recover with appropriate treatment effectively [1]. Many studies have been done in implementing classification and determining the presence of COVID-19 as well as in detecting the severity of pneumonia. CT and X-ray image modalities are non-invasive and used to detect and severity of lung infection [2,3]. In this study, we used principal component analysis (PCA) for feature

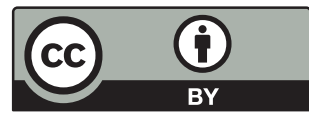

This work is licensed under a Creative Commons Attribution 4.0 International License, which permits unrestricted use, distribution, and reproduction in any medium, provided the original work is properly cited. 
extraction of CT images and a modified whale optimization algorithm (MWOA) for feature selection. To classify COVID-affected images from a large dataset and detect severity using the modified whale optimization algorithm (MWA) with the salp swarm algorithm (MWOA-SSA). The main disadvantage of existing algorithms are inefficiency, high execution time, and maximized error rate. To overcome these issues, our proposed MWOA-SSA has high potential in detecting the severity of lung infections such as pneumonia and classifying COVID-19 in affected and unaffected images from a large dataset effectively and quickly.

To predict coronavirus, X-ray images play a more important role than CT because the former is less sensitive. Furthermore, X-ray images are used to diagnose the early and mild stages of coronavirus patients. CT images are also is used in the diagnosis of coronavirus and improving efficiency in terms of dosage in radiation [4]. To enhance the improvement in scanning images in a sliced manner effectively by using multi-slice computerized tomography (MSCT) [5]. To achieve improvement in larger temporal resolution achieved by dual source CT image [6].

Machine learning algorithms have been used for the last decades in medical applications for computer-based diagnosis, helping physicians diagnose at earlier stages of diseases and providing better customized therapies to patients [7,8]. Approaches to find the best solution from all possible solutions of a particular radiology problem are known as meta-heuristic algorithms. The acceptable best solution of the optimization technique requires less computational effort within a stipulated time [9]. For the feature selection, the proposed MWOA is implemented with a binary optimizer in terms of average select size, error rate, mean, standard deviation, average fitness, best fitness, and worst fitness. The main contributions of this study are as follows,

1. A COVID-19 classification based on proposed algorithms for feature classification of WMOASSA is developed.

2. A novel approach in detecting severity of lung infection based on severity level is implemented.

3. The proposed WMOA-SSA can effectively classify the input CT images as COVID-19 or nonCOVID-19.

The paper has been organized as follows. Section 2 presents the literature review. Section 3 introduces the classification of COVID-19 images using MWOA-SSA. Section 4 discusses the experimented results. Section 5 concludes the paper and provides future directions.

\section{Review of Literature}

This section describes the recent literature on feature classification and prediction of coronavirus. COVID-19 has affected human beings in every aspect of their daily lives. To diagnosis the coronavirus disease by using various modalities of image such as CT and X-ray image. Through these images, physicians scan and diagnose at early stages and during disease progression. Many studies have been published on the prediction of coronavirus. Our aim is to achieve effectiveness in classifying COVID-19 case images from a large dataset and detect the severity of lung infections such as pneumonia. A previous paper [10] proposed evaluating the infection rate in CT scans of lungs using visual and coronal axes. By using visual inspection COVID-19 disease is used to identify the lung infection [11].

Another paper [12] proposed implementing a visual infection-based method to detect lung infection using lung CT scan. Authors in [13] implemented deep learning algorithms to identify and screen COVID-19 patients using the modality of CT images accurately. By using an artificial intelligence (AI) technique for diagnosis, COVID-19 patients are identified based on convolutional 
neural network (CNN) using CT slices images, helping accurately classify COVID-19 from nonCOVID-19 groups [14]. The machine learning algorithm fractional multichannel exponent moments method is used to extract features from the chest X-ray image and used to classify COVID-19 or nonCOVID-19 patients [15]. Tab. 1 shows a summary of recent research work in COVID-19.

Table 1: Survey on existing algorithms

\begin{tabular}{lll}
\hline Author name & Modality of image & Methods \\
\hline Hu et al. (2020) [16] & CT image & Supervised deep learning \\
Nour et al. (2020) [17] & X-Ray image & CNN, support vector machine (SVM) \\
Wu et al. (2020) [18] & CT image & ResNet50 based deep learning \\
Ardakani et al. (2020) [14] & CT image & ResNet-101 based deep learning \\
Zhang et al. (2020) [19] & CT image & AI based ResNet \\
Panwar et al. (2020) [20] & X-Ray image & Transfer learning, deep CNN \\
Butt et al. (2020) [21] & CT image & CNN \\
Al-Tashi et al. (2019) [22] & CT image & Hybrid grey wolf optimization \\
Ye et al. (2019) [23] & CT image & Adaptive statistical iterative \\
& & reconstruction-V technique \\
Yamashita et al. (2018) [24] & X-ray image & CNN \\
Fu et al. (2018) [25] & CT, X-Ray image & Multimodal CNN \\
Walker et al. (2017) [26] & CT, X-Ray image & Multimodal CNN \\
\hline
\end{tabular}

\section{Enhanced Whale with Salp Swarm Optimization Methodology}

This work introduces the concept of classification of affected lung disease and its severity. This proposed work has four stages. First, a median filter is used for pre-processing. Second, PCA is used for texture feature extraction. Third, A MWOA is used for selecting features. Fourth, the proposed MWOA-SSA is used for classification and identifying the severity. The architecture of our proposed method is given in Fig. 1. CT scan images are collected and preprocessed using a median filter. PCA is used to remove unwanted textures in the images. Then, the images are processed using MWOA-SSA to classify the affected image.

This proposed work consists of four phases:

Phase 1: Pre-processing using a median filter.

Phase 2: Feature extraction using PCA.

Phase 3: Feature selection using MWOA.

Phase 4: Proposed work on classification of infected lung images from a large dataset using MWOA-SSA. 


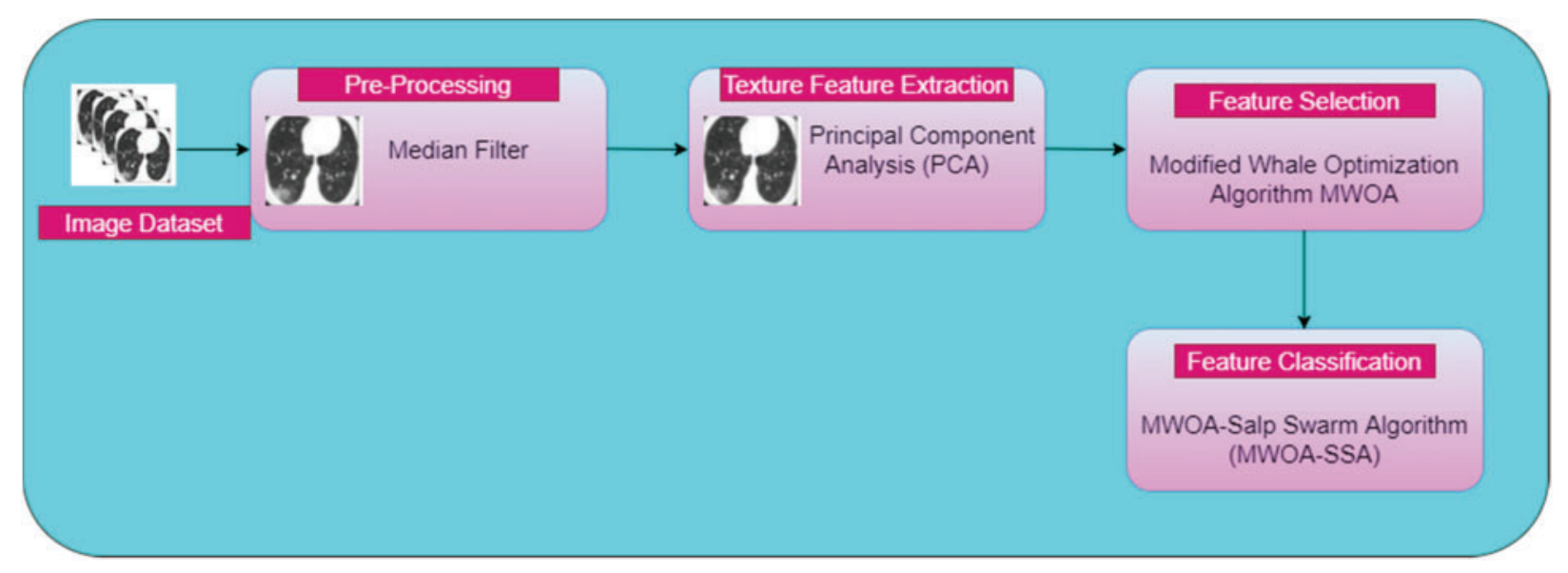

Figure 1: Architecture of proposed work

\subsection{Pre-Processing}

The aim of pre-processing is to improve the high quality of the CT scan chest image. We need to denoise the image by applying a median filter. This median filter scans the entire image using an $8 \times 8$ matrix and replaces the center pixel value by choosing the median of all pixel values inside the $8 \times 8$ matrix by using

$\operatorname{img}[a, b]=\operatorname{median}\{\operatorname{imgo}[i, j],\{i, j) \in y\}$

where $y$ is the neighborhood pixel value represented by the user and $i, j$ is the center pixel value's location.

\subsection{Texture Feature Extraction Using PCA}

The idea behind PCA is to map m-dimensional features to $\mathrm{n}$ dimensions that have a set of orthogonal feature values. Feature extraction using PCA meets the variance of sample pixel values after reduction of dimensionality and minimizes the error rate. The steps needed for texture feature extraction using PCA are given below, and Fig. 2. Provides an overview of PCA operation.

\section{Algorithm 1: Texture Feature Extraction using PCA}

Step 1: To standardize the original pixel values, subtract all sample pixel values from the mean value of corresponding feature value by using s $\mathrm{s}$

$\overline{A_{j}}=\frac{1}{n} \sum_{i=1}^{n} A_{i j}$

Step 2: Evaluate the covariance matrix $C\left(c=\left(A_{j k}\right)_{n \times n}\right.$ where $\mathrm{n}$ is the number of features; $A_{j k}$ is the correlation between $\mathrm{j}^{\text {th }}$ and $\mathrm{k}^{\text {th }}$ feature value, where $j=1,2, \ldots, n ; k=1,2, \ldots, n$.

$C=\left[\begin{array}{llll}A_{11} & A_{12} & \cdots & A_{1 n} \\ A_{21} & A_{22} & \cdots & A_{2 n} \\ \vdots & \vdots & \ddots & \vdots \\ A_{n 1} & A_{n 2} & \cdots & A_{n n}\end{array}\right]$ 


Algorithm 1: Continued
Step 3:

For the covariance matrix, calculate the eigen value of $\lambda_{i}$, and the eigenvector value is $e i_{i}$.

$\lambda_{i} e i_{i}=C e i_{i}$

Step 4: Store the output values of eigenvector from large to small values

$\lambda_{1} \geq \lambda_{2} \geq \ldots \lambda_{n}$ and calculate the rate of contribution for each principal component. The rate of contribution is given below:

$\frac{\lambda_{k}}{\sum_{k=1}^{n} \lambda_{k}}$

Step 5: Transform the original matrix $A$ into a new matrix $B\left(B=\left(B_{j k}\right)_{n \times n 1}\right.$, where $\mathrm{j}=1,2 \ldots \mathrm{n}$ and $\mathrm{k}=1,2, \ldots \mathrm{n}$.

$B=A \times f_{1}, f_{2}, \ldots, f_{n 1}$

where $f_{1}, f_{2}, \ldots, f_{n}$ denotes a new feature space which is composed of $\mathrm{n} 1$ vector feature values, and $\mathrm{n} 1$ is extracted features by PCA. Fig. 2 shows the working principle of PCA.

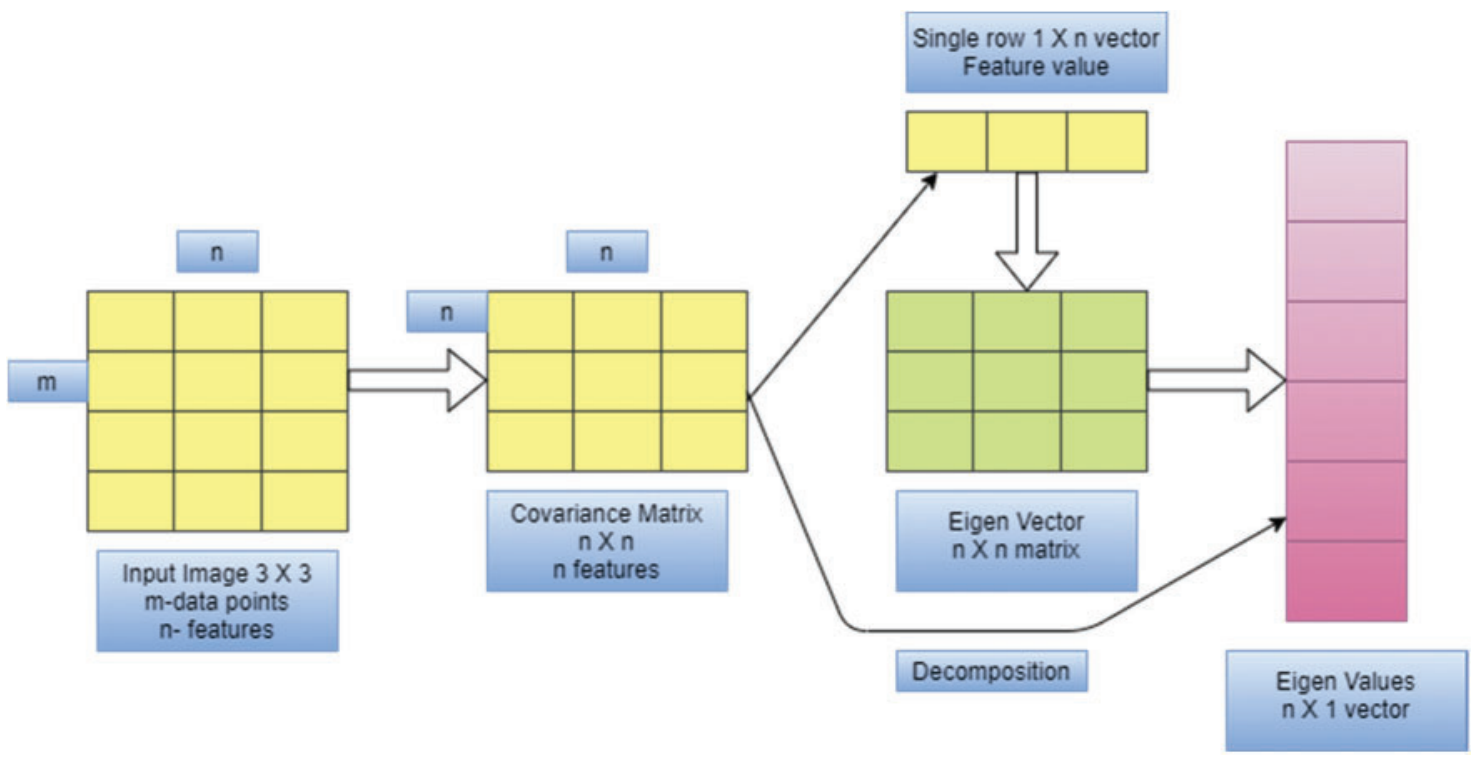

Figure 2: Overview of PCA

\subsection{Feature Selection Using $M W O A$}

Feature selection of brain image using MWOA, which is based on the behavior of whales, in which for trapping the prey bubbles are involved for searching in a spiral-shaped [27,28]. The whale is randomly selected, and it can be updated by the best whale value that gives the optimal solution.

$\vec{F}(n+1)=\overrightarrow{F_{\text {rand }}}-\vec{A} \cdot \vec{D}=\left|\vec{c} \cdot \overrightarrow{F_{\text {rand }}}-\vec{F}\right|$ 
To improve this result, the performance of three whales are randomly chosen, and it cannot be affected by the leader's position. Eq. (6) is modified as follows:

$\vec{F}(n+1)=\vec{w}_{1} * \vec{F}_{r n d 1}+\vec{x} * \vec{w}_{2} *\left(\vec{F}_{r n d 2}-\vec{F}_{r n d 3}\right)+(1-\vec{x}) * \vec{w}_{3} *\left(\vec{F}-\vec{F}_{r n d 1}\right)$

where, $\vec{F}_{r n d 1}, \vec{F}_{r n d 2}$ and $\vec{F}_{r n d 3}$ are randomly chosen solutions (prey). $\vec{w}_{1}$ is a random value between [0,0.5]. $\vec{w}_{2}$ and $\vec{w}_{3}$ are random values between $[0,1] . \vec{x}$ decreases the value and smoothens exploration and exploitation by using

$\vec{x}=1-\left(\frac{i t}{\operatorname{Max}_{i t}}\right)^{2}$

where $\mathrm{t}$ represents iteration number, and $\operatorname{Max}_{i t}$ represents the maximum number of iterations.

The algorithm is given as follows:

Algorithm 2: MWOA

Input: Lung Image

Output: Detecting COVID presence images

Step 1: Initialize Population $\vec{F}_{i}(i=1,2, \ldots n)$, maximum iteration max_it, function of fitness $F i_{n}$.

Step 2: Initialize parameters of WOA $\vec{B}, \vec{b}, \overrightarrow{c,} \overrightarrow{u_{1}}, \overrightarrow{u_{2}}, \overrightarrow{r_{3}}, h$ and modified parameters $\vec{w}_{1}, \vec{w}_{2}, \vec{w}_{3}$.

Step 3: Initialize $\mathrm{t}=1$.

Step 4: Convert output into binary values as 0 or 1 .

Step 5: Evaluate fitness value $F i_{n}$ for each $\vec{F}_{i}$.

Step 6: Find best individual value by $\overrightarrow{F^{*}}$.

Step 7: While $n \leq$ max_iter do

Step 8: For $i=(1 ; i<n+1)$ do

Step 9: If $\left(\overrightarrow{u_{3}}<0.5\right)$ then

Step 10: If $(|\vec{B}|<1)$ then

Step 11: Update current position of agent for search by using Eqs. (2), (3).

Step 12: Else

Step 13: Choose three search agents randomly $\vec{F}_{r n d 1}, \vec{F}_{r n d 2}, \vec{F}_{r n d 3}$.

Step 14: Update agent's exponential form by using Eq. (9).

Step 15: Update current position of agent for search in exponential form by using Eq. (8).

Step 16: End if

Step 17: Else

Step 18: Update current position of agent for search by using

$\vec{F}(t+1)=\overrightarrow{D^{\prime}} \cdot e^{b h} \cdot \cos (2 \pi h)+\vec{F}^{*}(t)$

Step 19: End if

Step 20: End For

Step 21: for $(i=1 ; i<n+1)$ do

Step 22: Evaluate $\overrightarrow{F_{i}^{*}}=\operatorname{Gaussian}\left(\mu \vec{F}^{*}, \sigma\right)+\left(\eta \times{\overrightarrow{F^{*}}}^{\prime}-\eta^{\prime} \times \vec{P}_{i}\right)$

Step 23: End For

Step 24: Update $\vec{B}, \vec{b}, \vec{c}, \overrightarrow{w_{3}}, h$ 


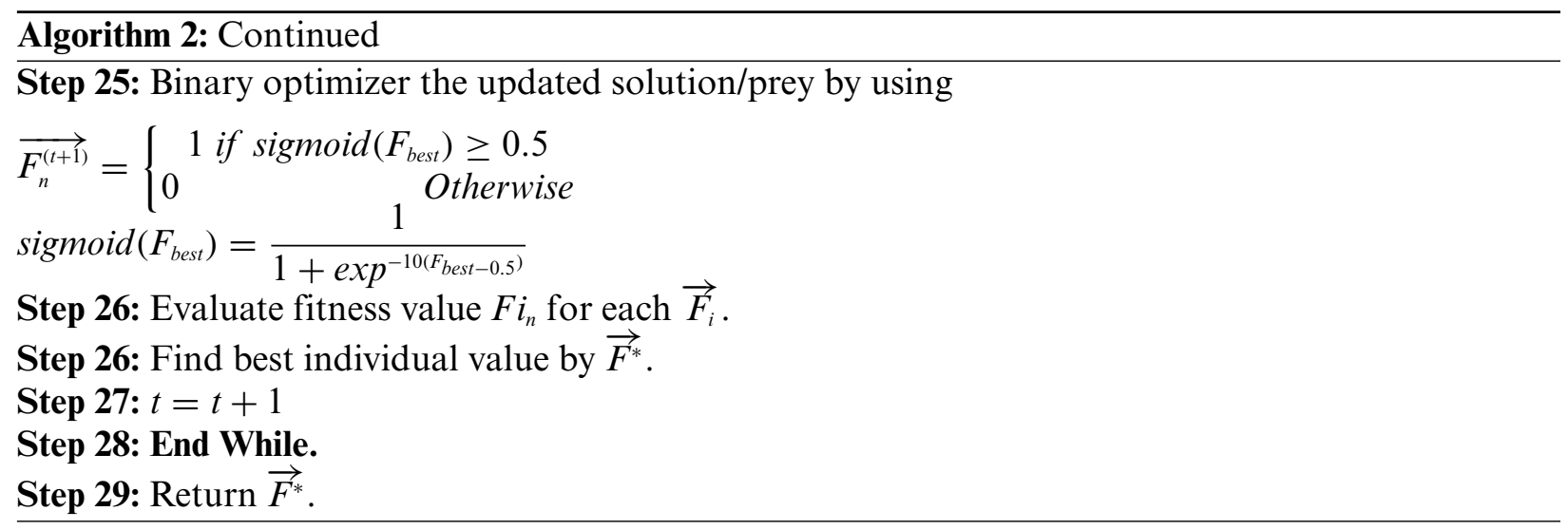

\subsection{Proposed Feature Classification Using MWOA-SSA}

In this phase, classification of infected lung images from a large dataset is done using MWOA-SSA. To improve the accuracy and optimal solution, the SSA) is used with MWOA. This SSA randomly initializes the swarm of $\mathrm{N}$ salps. The swarm is represented by the 2-D matrix mat. Searching food for swarm is represented as $s f$, and leader's movement in the form of a chain is denoted as $s x_{i}^{\prime}$. It is represented by using

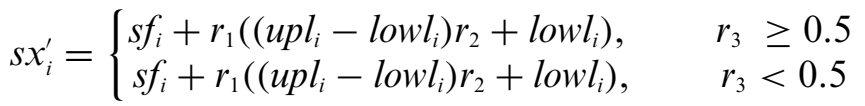

where $i$ is swarm's dimension position, and it is updated. $s f_{i}$ is the $i^{\text {th }}$ position for a source of food. $u p l_{i}$ and $l o w l_{i}$ are the upper and lower limits of the $i^{t h}$ element. $r_{1}$ is a dynamic variable for iteration. $r_{2}$ and $r_{3}$ are random numbers between $[0,1]$ calculated as

$r_{1}=2 e^{-\left(^{4 i t}\right.}{ }^{\left(\text {iter }^{2}\right.}$

where it represents the current iteration and iter is the maximum number of iterations. $r_{1}$ is a control variable that controls the balance between exploitation and exploration of the optimization algorithm. It is represented as

$s x_{i}^{\prime}=0.5\left(s x_{i}^{\prime}-s x_{i}^{i-1}\right)$

The procedure for SSA is given as follows.

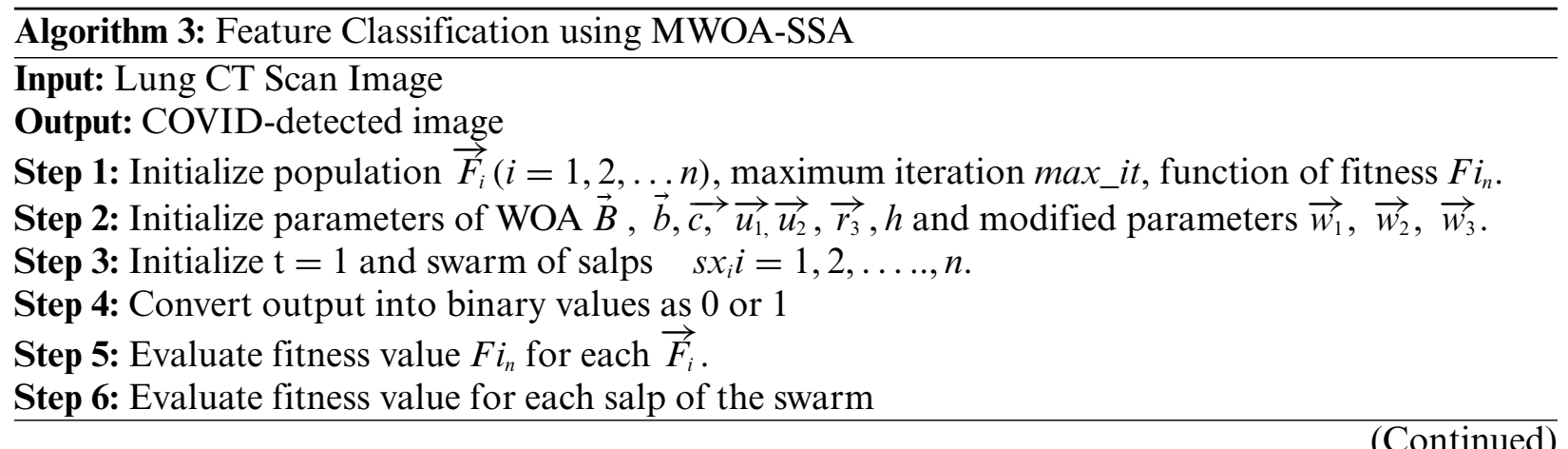




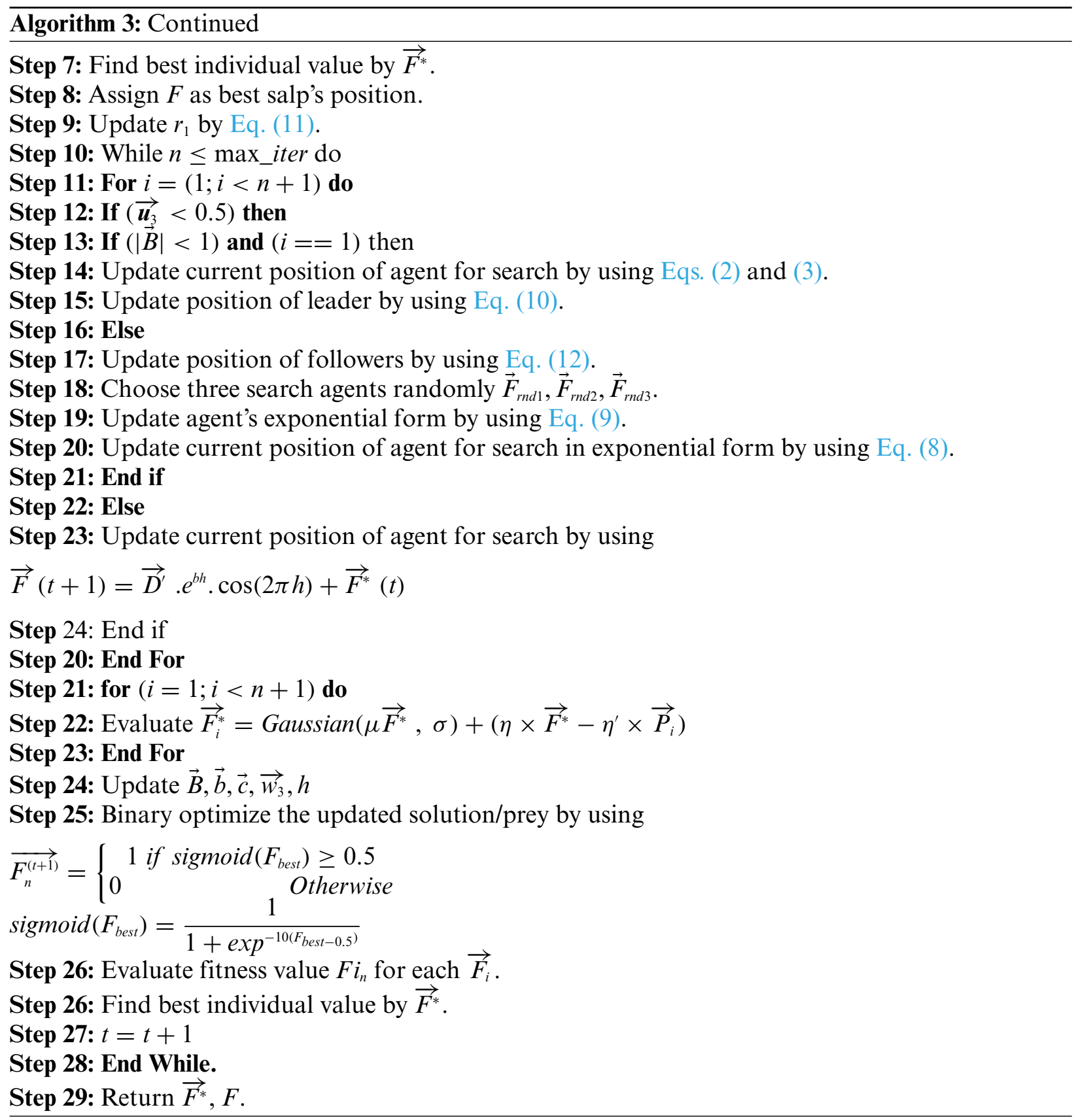

\section{Results and Analysis}

For the experimental result, data are collected from the Kaggle dataset [29], which has 1,500 CT images of COVID-19 and non-COVID 19. MWOA-SSA is compared with the existing algorithms MWOA [30] and SSA [31] by using performance metric measures of sensitivity, specificity, accuracy, precision (PPV), F-score, and negative predictive value (NPV).

TP - True Positive, TN - True Negative, FN - False Negative, FP - False Positive, 
These metric measures are defined by using:

\section{Sensitivity}

It is also called true positive rate or recall.

sensitivity $=\frac{T P}{T P+F N}$

\section{Specificity}

It is called true negative rate (TNR).

Specificity $=\frac{T N}{T N+F P}$

Accuracy

Accuracy $=\frac{T P+T N}{T P+T N+F P+F N}$

\section{Precision}

It is called positive predictive value (PPV).

$P P V=\frac{T P}{T P+F P}$

\section{Negative Predictive Value}

It evaluates true negatives for all negative values by using

$N P V=\frac{T N}{T N+F N}$

F-Score

It is used to measure sensitivity and mean of harmonic by using

$F-$ Score $=2 \times \frac{P P V \times T P R}{P P V+T P R}$

Tab. 2 shows the performance metric measures of feature extraction.

Table 2: Performance metric measures of feature extraction

\begin{tabular}{llllll}
\hline Algorithm & Sensitivity & Specificity & PPV & NPV & F-score \\
\hline PCA & $84.4 \%$ & $77.2 \%$ & $74.6 \%$ & $81.3 \%$ & $75.1 \%$ \\
MWOA & $95.2 \%$ & $89.5 \%$ & $82.1 \%$ & $85.2 \%$ & $83.2 \%$ \\
MWOA-SSA & $97.8 \%$ & $91.7 \%$ & $88.3 \%$ & $93.6 \%$ & $96.4 \%$ \\
\hline
\end{tabular}

Tab. 2 shows that for the sensitivity rate, MWOA-SSA is better than PCA (84.4\%) and MWOA (95.2\%). MWOA-SSA outperforms other algorithms with a specificity of $97.8 \%$. For PPV, MWOASSA has a percentage of $88.3 \%$. For NPV, MWOA-SSA has $93.6 \%$. MWOA-SAA outperforms other algorithms with an F-score of $96.4 \%$. 


\subsection{Feature Selection}

MWOA-SSA is used for feature selection, and it is compared with existing algorithms of PCA and MWOA in terms of average fitness, average error, best fitness, mean, standard deviation, and worst fitness. The parameter values for the fitness function are 0.97 and 0.03 .

\section{Average Error}

It shows the classifier's accuracy for the feature selection for the COVID-19 dataset, and it is calculated by using

AverageErr $=1-\frac{1}{R} \sum_{j=1}^{R} \frac{1}{S} \sum_{i=1}^{N} \operatorname{Comp}\left(C l_{i}, l b_{i}\right)$

where $C l_{i}$ is classifier's label for the pixel $i$ and $l b_{i}$ is the class label for the pixel $i$ of the image and Comp calculates the matching between two inputs.

Mean

Mean $=\frac{1}{R} \sum_{j=1}^{M} m e_{j}^{*}$

Standard Deviation

$S D=\sqrt{\frac{1}{N-1}} \sum^{\left(m e_{j}^{*}-m e a n\right)^{2}}$

where mean is obtained from Eq. (17)

\section{Best Fitness}

It calculates the minimum function of fitness, and it is calculated as

Bestfit $_{n}=\operatorname{Min}_{j=1}^{M} m e_{j}^{*}$

\section{Average Fitness}

The average size of features in the COVID-19 dataset is calculated as

Avgsize $=\frac{1}{M} \sum_{j=1}^{M} \frac{\operatorname{size}\left(m e_{j}^{*}\right)}{D}$

\section{Worst Fitness}

The worst solution of fitness is calculated as

worstfit $_{n}=\operatorname{Max}_{j=1}^{M} m e_{j}^{*}$

Tab. 3 shows the performance of the proposed algorithm in feature selection

The results of the proposed MWOA-SSA algorithm in Tab. 3 show the lower error and select features from the COVID-19 dataset. The MWOA-SSA algorithm achieved the minimum average error of 0.1114 in selecting the features of infected lung images. The minimum errors for PCA, MWOA, and MWOA-SSA are used to select the features from best fitness to worst fitness. The proposed algorithm MWOA-SSA outperforms other existing algorithms, and the best fitness value is 0.1034 , the worst fitness value is 0.2115 , and the average fitness value is 0.2034 . 
Table 3: Performance metric measures for feature selection

\begin{tabular}{llll}
\hline Optimizer & PCA & MWOA & MWOA-SSA \\
\hline Average error & 0.1652 & 0.1547 & 0.1114 \\
Average select size & 0.3234 & 0.3548 & 0.0715 \\
Mean & 0.3452 & 0.4134 & 0.1573 \\
Standard deviation & 0.0367 & 0.0678 & 0.0123 \\
Best fitness & 0.1264 & 0.1598 & 0.1034 \\
Worst fitness & 0.2763 & 0.2356 & 0.2115 \\
Average fitness & 0.2287 & 0.2419 & 0.2034 \\
\hline
\end{tabular}

\subsection{Detection and Severity Classification of COVID-19}

To detect the infection severity, lung images have been examined by using ground truths of CT0CT4 as given below. Tab. 4 presents the severity levels in the lungs.

Table 4: Severity levels for infection in lungs [32,33]

\begin{tabular}{ll}
\hline Class & Infection in $\%$ \\
\hline Healthy & 0 \\
Mild & $1-25 \%$ \\
Moderate & $26-50 \%$ \\
Severe & $51-75 \%$ \\
Critical & $76-100 \%$ \\
\hline
\end{tabular}

In this work, we collected data on 500 patients with COVID-19 infection. Infection was confirmed by a nasopharyngeal swab using a U-TOP COVID-19 Detection Kit. Age, gender, d-dimer, ferritin levels, C-reactive protein test (CRP), and O2 were collected. Patient's age was classified into $<20,21-$ $40,41-49,50-60,61-70$, and $>70$ years. The correlation $(p<0.05)$ between CT severity score was used to detect lung infection. Tab. 5 shows a survey of 500 patients who are affected by pneumonia. Fig. 3. shows the CT severity of COVID-19 patients.

Table 5: Demographic data of 500 patients

\begin{tabular}{lll}
\hline Age in years & Male (300 patients) & Female (200 patients) \\
\hline 5 to 20 & 53 & 40 \\
21 to 40 & 87 & 35 \\
41 to 60 & 65 & 45 \\
61 to 70 & 35 & 35 \\
More than 71 years & 60 & 45 \\
\hline
\end{tabular}

Fig. 3 shows that negative disease was mainly seen in the age group of 21 to 40 (30\%), mild lung mainly infection was seen in the 41 to 60 age group (60\%). Moderate lung infection was mainly seen 
in the 61 to 70 age group (68\%), and severe lung infection was mainly seen in the age group of 41 to $60(70 \%)$. This is the highest risk factor for COVID-19 affected patients [34-37]. Fig. 4. shows the time taken for the classification of COVID-19 affected cases and non-COVID-19 cases from the large dataset.

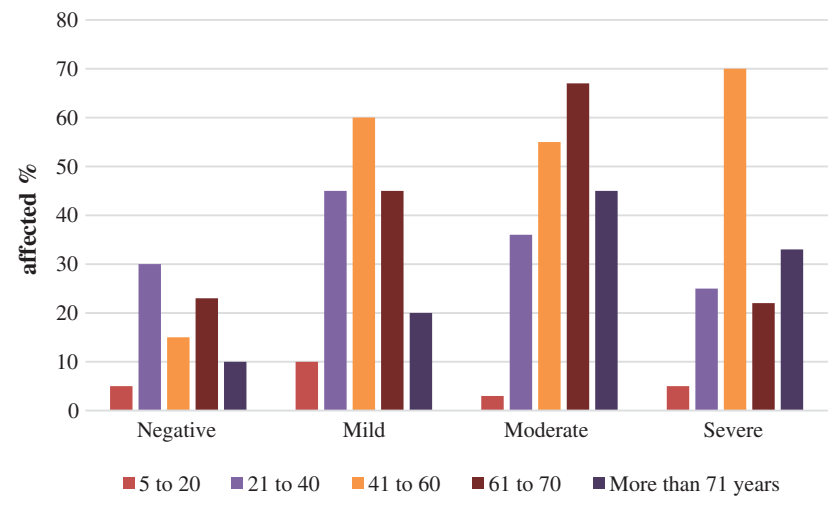

Figure 3: CT-COVID severity score

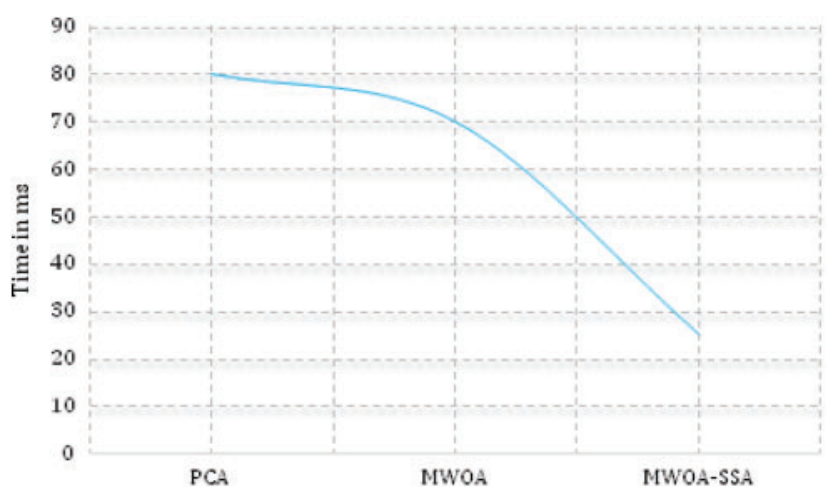

Figure 4: Execution time (proposed method executes faster than PCA and MWOA)

\section{Conclusion}

MWOA-SSA is used for the classification of COVID-19 cases in four phases. In the first phase, to classify accurate COVID-19 and non-COVID-19 images from a large dataset, pre-processing work has been done using a median filter. Features are extracted for the training CT images by PCA. For the feature selection of CT lung images, MWOA is implemented. For the selected features of the CT image, MWOA-SSA is implemented to classify the COVID-19 and non-COVID-19 images from the large dataset. This paper also proposes detecting and identifying the severity of lung infection by using different severity levels of COVID-19 cases. The main advantage of MWOA-SSA is that it efficiently and quickly classifies COVID-19 and non-COVID-19 cases and detects severity of lung infection using severity levels. MWOA-SSA has an accuracy of $97 \%$, whereas PCA and MWOA have accuracies of $81 \%$ and $86 \%$. In future work, we suggest the use of various deep learning algorithms and various modalities of images and clinical reports.

Funding Statement: The authors received no specific funding for this study. 
Conflicts of Interest: The authors declare that they have no conflicts of interest to report regarding the present study.

\section{References}

[1] Q. V. Pham, D. C. Nguyen, T. Huynh, W. J. Hwang and P. N. Pathirana, "Artificial Intelligence (AI) and big data for coronavirus (COVID-19) pandemic: A survey on the state-of-the-arts," IEEE Access, vol. 8, pp. 130820-130839, 2020.

[2] X. H. yuen Frank Wong, H. Yin Sonia Lam, A. Ho Tung Fong, S. Leung, T. Wing Yan Chin et al., "Frequency and distribution of chest radiographic findings in patients positive for COVID-19," Radiology, vol. 296, no. 2, pp. E72-E78, 2020.

[3] C. Sanghoon, L. Sunho, K. Changhwan, W. Sunhee, K. Taejin et al., "Enhancement of soft-tissue contrast in cone-beam CT using an anti-scatter grid with a sparse sampling approach," Physics in Medicine and Biology, vol. 7, pp. 1-9, 2020.

[4] B. J. Walker, J. Radtke, G. H. Chen, K. W. Eliceiri and T. R. Mackie, "A beam optics study of a modular multi-source X-ray tube for novel computed tomography applications," Nuclear Instruments and Methods in Physics Research Section A: Accelerators, Spectrometers, Detectors and Associated Equipment, vol. 868, pp. 1-9, 2017.

[5] L. T. Campos, F. M. Jesus, E. A. De Souza Gonçalves and L. A. G. Magalhães, "Computed tomography x-ray characterization: A monte carlo study," Radiation Physics and Chemistry, vol. 167, Article ID 108359, 2020.

[6] M. K. Honkanen, H. Matikka, J. T. Honkanen, A. Bhattarai, A. Grinstaff et al., "Imaging of proteoglycan and water contents in human articular cartilage with fullbody CT using dual contrast technique," Journal of Orthopaedic Research, vol. 37, no. 5, pp. 1059-1070, 2019.

[7] E. Montagnon, M. Cerny, A. Cadrin chánevert, V. Hamilton, T. Derennes et al., "Deep learning workflow in radiology: A primer," Insights into Imaging, vol. 11, no. 1, Article Number 22, 2020.

[8] A. Ibrahim, S. Mohammed, H. A. Ali and S. E. Hussein, "Breast cancer segmentation from thermal images based on chaotic salp swarm algorithm," IEEE Access, vol. 8, no. 1, pp. 122121-122134, 2020.

[9] M. A. A. Qaness, A. A. Ewees, H. Fan and M. Abd, "Optimization method for forecasting confirmed cases of COVID-19 in China," Journal of Clinical Medicine, vol. 9, no. 3, Article Number 674, 2020.

[10] K. Li, Y. Fang, W. Li, C. Pan, P. Qin et al., "CT image visual quantitative evaluation and clinical classification of coronavirus disease (COVID-19)," European Radiology, vol. 30, no. 8, pp. 4407-4416, 2020.

[11] M. Chung, A. Bernheim, X. Mei, N. Zhang, M. Huang et al., "CT imaging features of 2019 novel coronavirus (2019-nCoV),"Radiology, vol. 295, no. 1, pp. 202-207, 2020.

[12] R. Yang, X. Li, H. Liu, Y. Zhen, X. Zhang et al., "Chest CT severity score: An imaging tool for assessing severe COVID-19," Radiology Cardiothoracic Imaging, vol. 2, no. 2, pp. e200047, 2020.

[13] X. Wu, H. Hui, M. Niu, L. Li, L. Wang et al., "Deep learning-based multi-view fusion model for screening 2019 novel coronavirus pneumonia: A multicentre study," European Journal of Radiology, vol. 128, Article ID $109041,2020$.

[14] A. A. Ardakani, A. R. Kanafi, U. R. Acharya, N. Khadem and A. Mohammadi, "Application of deep learning technique to manage COVID-19 in routine clinical practice using CT images: Results of 10 convolutional neural networks," Computers in Biology and Medicine, vol. 121, Article ID 103795, 2020.

[15] M. A. Elaziz, K. M. Hosny, A. Salah, M. M. Darwish, S. Lu et al., "New machine learning method for image-based diagnosisof COVID-19," PLoS ONE, vol. 15, no. 6, p. e0235187, 2020.

[16] S. Hu, Y. Gao, Z. Niu, Y. Jiang, L. Li et al., "Weakly supervised deep learning for COVID-19 infection detection and classification from CT images," IEEE Access, vol. 8, pp. 118869-118883. 2020.

[17] M. Nour, Z. Cömert and K. Polat, "A novel medical diagnosis model for COVID-19 infection detection based on deep features and Bayesian optimization," Applied Soft Computing, vol. 97, Part A, p. 106580, 2020. 
[18] S. Hu, Y. Gao, Z. Niu, Y. Jiang and L. Li, "Weakly supervised deep learning for COVID-19 infection detection and classification from CT images," IEEE Access, vol. 8, pp. 118869-118883, 2019.

[19] K. Zhang, X. Liu, J. Shen, Z. Li and Y. Sang, "Clinically applicable AI system for accurate diagnosis, quantitative measurements, and prognosis of COVID-19 pneumonia using computed tomography," Cell, vol. 181, no. 6, pp. 1423-1433, 2020.

[20] H. Panwar, P. K. Gupta, M. K. Siddiqui, R. Morales Menendez and V. Singh, "Application of deep learning for fast detection of COVID-19 in X-rays using nCOVnet," Chaos, Solitons Fractals, vol. 138, Article ID $109944,2020$.

[21] X. Xu, X. Jiang, C. Ma, P. Du, X. Li et al., "A deep learning system to screen novel coronavirus disease 2019 pneumonia," Engineering, vol. 6, no. 10, pp. 1122-1129, 2020.

[22] Q. AlTashi, S. J. Abdul Kadir, H. M. Rais, S. Mirjalili and H. Alhussian, "Binary optimization using hybrid grey wolf optimization for feature selection," IEEE Access, vol. 7, pp. 39496-39508, 2019.

[23] K. Ye, Q. Zhu, M. Li, Y. Lu and H. Yuan, "A feasibility study of pulmonary nodule detection by ultralowdose CT with adaptive statisticaliterative reconstructionV technique," European Journal of Radiology, vol. 119, Article ID 108652, 2019.

[24] R. Yamashita, M. Nishio, R. K. G. Do and K. Togashi, "Convolutional neural networks: An overview and application in radiology," Insights into Image Processing, vol. 9, no. 4, pp. 611-629, 2018.

[25] J. Fu, J. Wang, W. Guo and P. Peng, "Multi-mounted X-Ray beam computed tomography," Nuclear Instruments and Methods in Physics Research, vol. 888, pp. 119-125, 2018.

[26] B. J. Walker, J. Radtke, G. H. Chen, K. W. Eliceiri and T. R. Mackie, "A beam optics study of a modular multi-source X-ray tube for novel computed tomography applications," Nuclear Instruments and Methods in Physics Research, vol. 868, pp. 1-9, 2017.

[27] S. Mirjalili and A. Lewis, "The whale optimization algorithm," Advances in Engineering Software, vol. 95, pp. 51-67, 2016.

[28] S. Mirjalili, S. M. Mirjalili, S. Saremi and S. Mirjalili, "Whale optim. algorithm: Theory, literature review, and application in designing photonic crystal filters," Studies in Computational Intelligence, vol. 811, Springer, pp. 219-238, 2020.

[29] E. S. E. Kenawy, M. Ibrahim, A. Mirjalili, S. Eid and M. M. Hussein, "Novel feature selection and voting classifier algorithms for COVID-19 classification in CT images," IEEE Access, vol. 8, pp. 179317179335,2020 .

[30] Z. M. Yaseen, H. Faris and N. Al-Ansari, "Hybridized extreme learning machine model with salp swarm algorithm: A novel predictive model for hydrological application," Complexity, vol. 2020, Article ID $8206245,2020$.

[31] G. A. Saeed, W. Gaba, A. Shah, A. Al Helali, E. Raidullah et al., "Correlation between chest CT severity scores and the clinical parameters of adult patients with COVID-19 pneumonia," Radiology Research and Practice, vol. 2021, Article ID 6697677, 2020.

[32] Y. Qiblawey, A. Tahir, M. E. Chowdhury, A. Khandakar and S. Kiranyaz, "Detection and severity classification of COVID-19 in CT images using deep learning," Diagnostics, vol. 11, no. 5, Article Number 893, 2021.

[33] J. Zhao, Y. Zhang, X. He and P. Xie, "COVID-CT-dataset: A CT scan dataset about COVID-19," arXiv preprint arXiv: 2003.13865, vol. 490, 2020.

[34] S. Kukan, S. Gokul, S.S. Vishnu Priyan, S. Barathi Kanna and E. Prabhu, "COVID-19: Smart shop surveillance system," in the Intelligent Sustainable Systems, 1st ed., vol. 1. Henderson, Singapore: Springer. 2022.

[35] S. Aruul Mozhi Varman, A. R. Baskaran, S. Aravindh and E. Prabhu, "Deep learning and IoT for smart agriculture using WSN," in Proc. ICCIC, Coimbatore, India, pp. 1-6, 2017.

[36] S. Kanakaprabha and D. Radha, "Analysis of COVID-19 and pneumonia detection in chest X-ray images using deep learning," in Proc. ICCISC, Idukki, India, pp. 1-6, 2021.

[37] H. Sathyan and J. V. Panicker, "Lung nodule classification using deep convnets on CT images," in Proc. ICCCNT, Bengaluru, India, pp. 1-5, 2018. 